\title{
Supervised Classification of Ventricular Abnormal Potentials in Intracardiac Electrograms
}

\author{
Giulia Baldazzi ${ }^{1,2, *}$, Marco Orrù ${ }^{2}$, Mirko Matraxia $^{3}$, Graziana Viola $^{4}$, Danilo Pani $^{2}$ \\ ${ }^{1}$ Department of Informatics, Bioengineering, Robotics and Systems Engineering (DIBRIS), \\ University of Genova, Genova, Italy \\ ${ }^{2}$ Department of Electrical and Electronic Engineering (DIEE), University of Cagliari, Cagliari, Italy \\ ${ }^{3}$ Medical Concept Lab, Sassari, Italy \\ ${ }^{4}$ Division of Cardiology, San Francesco Hospital, Nuoro, Italy
}

\begin{abstract}
Ventricular abnormal potentials (VAPs) identification is a challenging issue, since they constitute the ablation targets in substrate-guided mapping and ablation procedures for ventricular tachycardia (VT) treatment.

In this work, two approaches for the supervised classification of VAPs in bipolar intracardiac electrograms are evaluated and compared. To this aim, 954 bipolar electrograms were retrospectively annotated by an expert cardiologist. All signals were acquired from six patients affected by post-ischemic VT by the CARTO3 system at the San Francesco Hospital (Nuoro, Italy) during routine procedures.

The first classification approach was based on a support vector machine trained and tested on four different features, extracted from both the time and time-scale domain, to identify physiological and abnormal potentials. Conversely, in order to assess the significance of the first approach and its features, in the second approach all the samples constituting a time-domain segment of each bipolar electrogram were given as input to a feed-forward artificial neural network.

In both cases, the accuracy in VAPs and physiological potentials identification exceeded 79\%, suggesting their efficacy and the possibility of VAPs automatic recognition without identifying peculiar features.
\end{abstract}

\section{Introduction}

Among ventricular arrhythmias, ventricular tachycardia (VT) is frequently associated with sudden cardiac death, thus representing one important factor of increased risk of mortality. For these patients, although implantable cardioverter-defibrillators can be effective to terminate the VT, they cannot avoid its onset [1]. Substrate-guided mapping and catheter ablation in sinus rhythm (SR) represent one of the most successful treatments for VT [2], [3]. These clinical procedures aim at identifying slow conduction areas within the damaged myocardial substrate in SR, which are characterized by electrograms affected by ventricular abnormal potentials (VAPs), in order to silence them through catheter ablation [2], [3]. Specifically, the arrhythmia substrate of sustained VT is mostly identified in scar-related re-entrant circuits, which can be caused by prior myocardial infarction or other non-ischemic causes [1]. However, besides relying on the same principle, many different strategies for substrate-based ablation have been developed [4].

In the clinical practice, different 3D mapping systems are adopted by expert operators in order to recognize VAPs during substrate-guided mapping and ablation procedures, such as EnSite Precision (Abbott, Chicago, IL, USA), CARTO3v6, (Biosense Webster, Diamond Barr, CA, USA) and Rhythmia HDx (Boston Scientific, Cambridge, MA, USA). Since ablation outcomes strongly depend on the identification of VAPs, several supporting mapping tools and identification algorithms have been recently proposed, such as the CARTO3 Ripple Mapping Module [5], the simultaneous amplitude frequency electrogram transformation mapping [6], the Ensite Precision fractionation map [7], the Lumipoint algorithm [8], the fragmentation map [9] and the combination of voltage limit adjustment with the Fast Fourier Transform [10]. However, all these algorithms base the VAPs recognition on the adoption of thresholds empirically defined on specific indexes, without the adoption of machine learning tools.

On the basis of our previous findings [11], in this work a comparison of two different approaches to the supervised classification of VAPs and physiological potentials is presented, aiming to support operators in the identification of VAPs during ablation procedures. Both approaches were evaluated on a dataset composed of 954 beats from six subjects affected by post-ischemic VT.

In the first approach, two time-scale features by the 
continuous wavelet transform (CWT) and two timedomain features were extracted to train a support vector machine (SVM) classifier. In the second approach, in order to assess the significance of the first method and the actual need of exploiting features to solve the classification problem, an artificial neural network (ANN) trained and tested on time-domain segments of the same intracardiac potentials was implemented.

\section{Materials and Methods}

\subsection{SVM-based approach}

In the first method [11], four features (Pearson's correlation coefficient below 0.83 ) from the time-scale and time domain were identified for the automatic recognition of VAPs.

Initially, in order to remove noisy oscillations of the signal that could affect the subsequent feature extraction, a translation-invariant wavelet denoising was performed on each intracardiac electrogram [11]. Then, from each denoised electrogram, a $350 \mathrm{~ms}$ window, including $50 \mathrm{~ms}$ before and $300 \mathrm{~ms}$ after the reference annotation, was selected. On this window, the CWT decomposition was computed considering all the scales covering the signal spectral components between about $16 \mathrm{~Hz}$ and $500 \mathrm{~Hz}$. Then, the sum and standard deviation of the average powers on the five most powerful scales were considered as time-scale domain features.

As regards the time-domain features, the peak-to-peak amplitude of each bipolar electrogram and a fragmentation measure were considered, the former emphasizing the high voltage signals $(>1.5 \mathrm{mV})$ with respect to those originating from scar-related areas, while the latter to highlight fragmented electrograms typically associated with slowconduction areas.

Finally, a SVM classifier with radial basis function (RBF) kernel, $v$ set to 0.5 and box constraint to 1 , was trained and tested with these features.

\subsection{ANN-based approach}

In the second classification approach, the same timewindow of $350 \mathrm{~ms}$ around the reference point was adopted and the samples composing the time-domain course of each raw intracardiac electrogram were considered as input for an ANN classifier.

For this purpose, a pattern recognition feedforward network composed of one hidden layer of ten neurons was adopted with the Levenberg-Marquardt back-propagation algorithm.

\subsection{Dataset}

In order to carry out the assessment, a real dataset composed of 954 bipolar intracardiac electrograms was adopted. In Figure 1 an example of bipolar intracardiac electrograms of the dataset is shown.

Bipolar electrograms were manually annotated by an expert cardiologist as VAPs or physiological potentials. The manual labelling was performed in a retrospective analysis by looking at the intracardiac signals morphologies and timings with respect to the corresponding simultaneous surface ECG recordings.

Signals were acquired from six patients affected by post-ischemic VT at the San Francesco Hospital (Nuoro, Italy) between 2017 and 2018 during electroanatomic mapping in SR. Bipolar electrograms recordings constituting this dataset was performed by the CARTO3 system at a sampling frequency of $1 \mathrm{kHz}$ with an embedded band-pass filter between $16 \mathrm{~Hz}$ and $500 \mathrm{~Hz}$. Usual clinical protocols were adopted for the subsequent radiofrequency catheter ablation.

As in [11], only the ending portion of each 2.5 s-long recording was exploited for the classification. In this regard, the annotation of the reference point, automatically saved by the CARTO3 acquisition system, easily allowed the identification of each beat of interest.
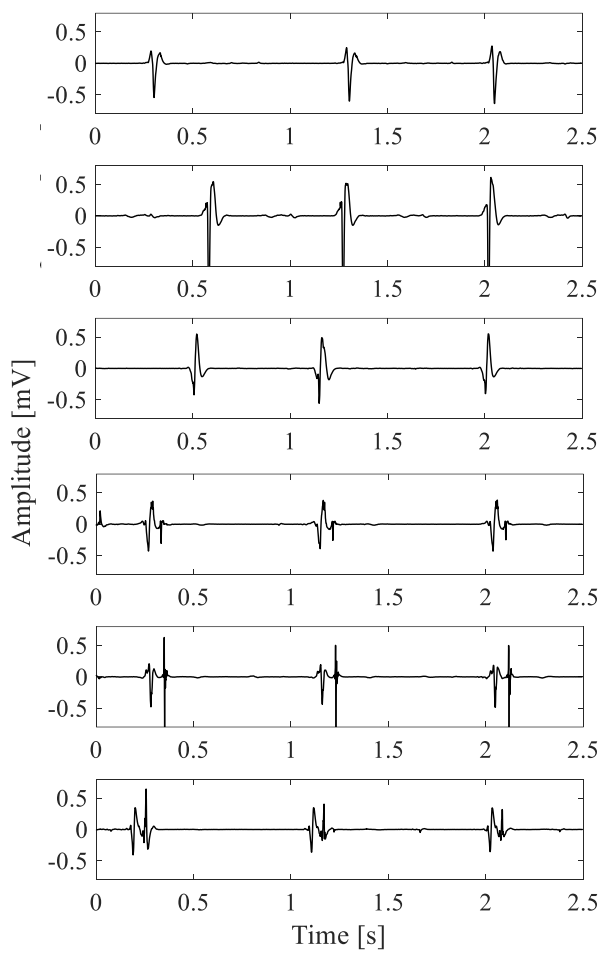

Figure 1: Examples of bipolar intracardiac electrograms constituting the real dataset adopted in this work.

\subsection{Performance evaluation}

In order to provide an objective quantitative evaluation of the proposed approaches, both classification methods were trained and tested with a 10-times 10-fold cross- 
validation scheme. Specifically, stratified partitions were adopted following the class proportions in the dataset (i.e., around $64 \%$ of normal beats and $36 \%$ of VAPs) to avoid discarding physiological examples or introducing unreal VAPs by means of oversampling methods. For each fold, $90 \%$ of the samples were divided into $80 \%$ for training and $10 \%$ for validation. The remaining $10 \%$ was used for test. Four performance indexes were computed: the accuracy (ACC), the True Positive Rate (TPR), the True Negative Rate (TNR) and the Positive Predictive Value (PPV):

$$
\begin{gathered}
\mathrm{ACC}=\frac{\mathrm{TP}+\mathrm{TN}}{\mathrm{P}+\mathrm{N}} \\
\mathrm{TPR}=\frac{\mathrm{TP}}{\mathrm{P}} \\
\mathrm{TNR}=\frac{\mathrm{TN}}{\mathrm{N}} \\
\mathrm{PPV}=\frac{\mathrm{TP}}{\mathrm{TP}+\mathrm{FP}}
\end{gathered}
$$

where $\mathrm{P}$ and $\mathrm{N}$ represent the abnormal and physiologic class sizes, TP and TN the number of abnormal and physiologic potentials correctly identified, respectively, while FP the normal electrograms classified as VAPs.

\section{Results}

In Table 1, the mean $(\mu)$ and standard error of the mean (s.e.m) computed across the 10-times 10-fold crossvalidation are reported for each performance index and classification approach.

The accuracy results point out that the classification performances are quite high and stable for both proposed methods. However, focusing on the percentages of TPR and TNR, their values are rather different. These findings suggest that the recognition of abnormal and physiological classes is not well-balanced. It is mainly true for the ANN classifier, which manages to recognize the physiological observations more correctly compared to the abnormal ones (i.e., significantly higher TNR than TPR). On the

Table 1. Mean and standard error for all performance indexes across the 10-times 10-fold cross-validation tests.

\begin{tabular}{l|cccc} 
Index & ANN & ANN & SVM & SVM \\
& $\mu$ & s.e.m & $\mu$ & s.e.m \\
\hline Acc[\%] & $79.3 \%$ & $1.5 \%$ & $79.4 \%$ & $1.0 \%$ \\
TPR[\%] & $62.1 \%$ & $1.8 \%$ & $71.7 \%$ & $1.4 \%$ \\
TNR[\%] & $89.2 \%$ & $1.3 \%$ & $83.8 \%$ & $1.3 \%$ \\
PPV[\%] & $77.6 \%$ & $2.2 \%$ & $72.1 \%$ & $1.9 \%$ \\
\hline
\end{tabular}

other hand, same conclusions can be drawn for the SVMbased approach, which however classifies the two classes in a slightly more homogeneous way. Nonetheless, PPV values underline that both approaches exhibit high precision.

For the sake of completeness, the confusion matrices for the best and worst cases identified for each classification method are reported in Figures 2 and 3.

\section{Conclusions}

In this work, a comparison between two different classification methods for the automatic identification of VAPs in intracardiac bipolar electrograms was presented. The first approach was based on a SVM classifier trained on time-scale domain and time domain features, while the other one on an ANN trained on the time-course of each
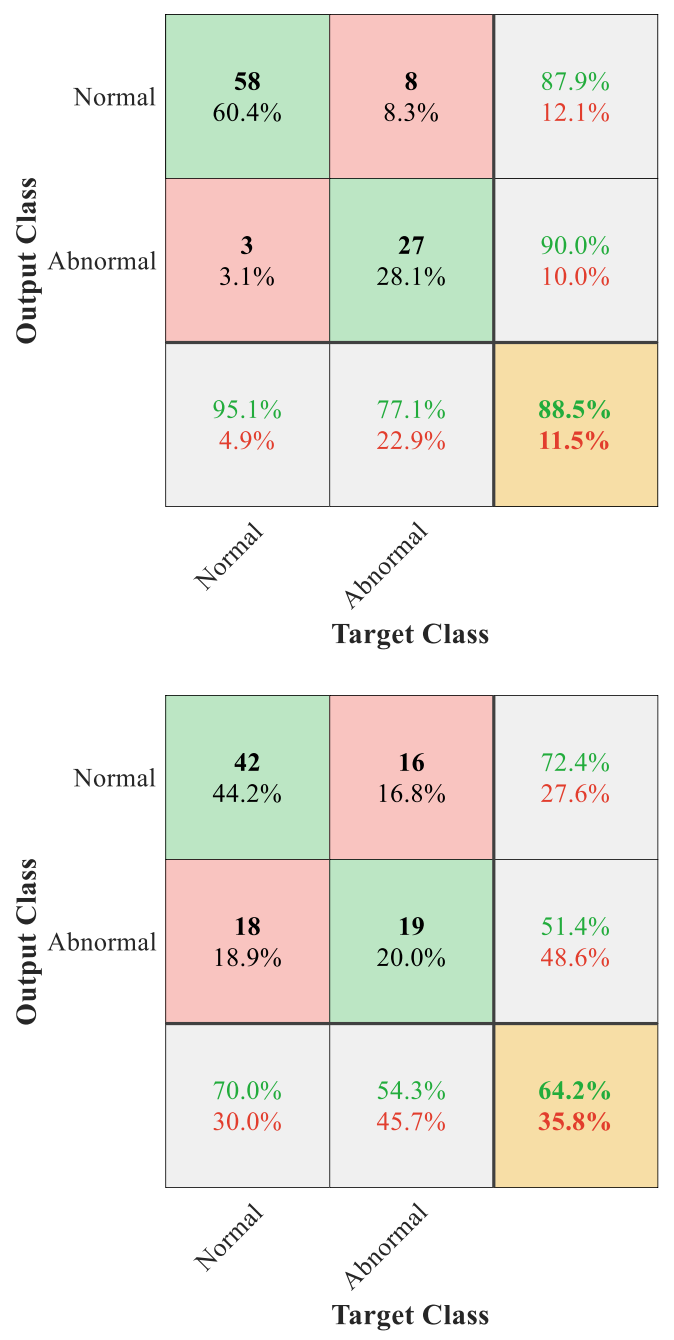

Figure 2: Confusion matrix related to the best (top) and worst (bottom) accuracy results obtained with the ANN approach over the 10-times 10-fold cross-validation. 

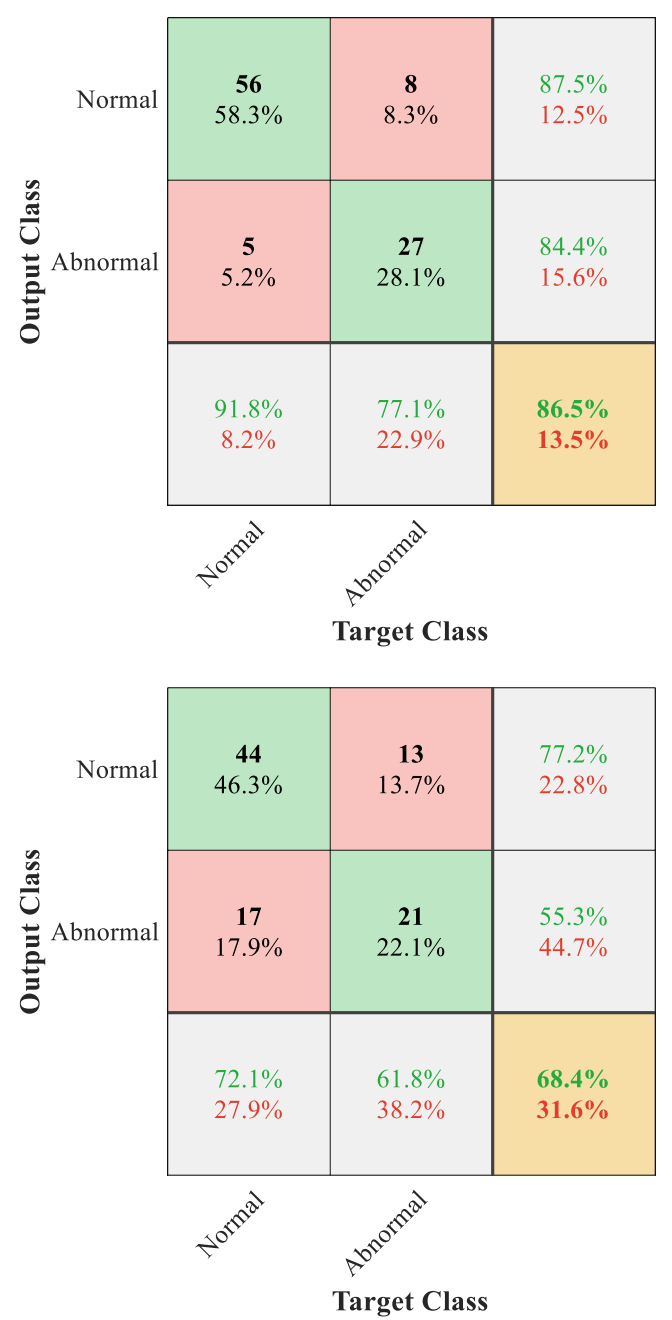

Figure 3: Confusion matrix related to the best (top) and worst (bottom) accuracy results obtained with the SVM approach over the 10-times 10-fold cross-validation.

bipolar recording. The latter was introduced in order to assess the actual need of exploiting features in the automatic VAPs recognition. Apart from our previous investigation, to the best of the authors' knowledge, this is the first time that artificial intelligence tools for VAPs recognition have been exploited.

Classification results showed high accuracy for both proposed methods, with some differences in terms of false positive and negative rates according to the chosen identification approach. These findings, which underline the possibility of an automatic recognition of VAPs also without trying to identify some peculiar features characterizing abnormal and physiological potentials, are promising. Due to the limited number of patients, a validation on a larger dataset will be pursued.

\section{Acknowledgment}

The authors wish to thank Ms. Giorgia Ibba for the collaboration in the development of the annotated dataset.

\section{References}

[1] E. Wissner, W. G. Stevenson, and K.-H. Kuck, "Catheter ablation of ventricular tachycardia in ischaemic and nonischaemic cardiomyopathy: where are we today? A clinical review," Eur. Heart J., vol. 33, no. 12, pp. 1440-1450, 2012.

[2] P. Vergara et al., "Late Potentials Abolition as an Additional Technique for Reduction of Arrhythmia Recurrence in Scar Related Ventricular Tachycardia Ablation," J. Cardiovasc. Electrophysiol., vol. 23, no. 6, pp. 621-627, Jun. 2012.

[3] P. Jaïs et al., "Elimination of local abnormal ventricular activities: a new end point for substrate modification in patients with scar-related ventricular tachycardia," Circulation, vol. 125, no. 18, pp. 2184-2196, 2012.

[4] D. F. Briceño et al., "Substrate ablation of ventricular tachycardia: late potentials, scar dechanneling, local abnormal ventricular activities, core isolation, and homogenization," Card. Electrophysiol. Clin., vol. 9, no. 1, pp. 81-91, 2017.

[5] L. Vishal et al., "A Prospective Study of Ripple Mapping the Post-Infarct Ventricular Scar to Guide Substrate Ablation for Ventricular Tachycardia," Circ. Arrhythmia Electrophysiol., vol. 9, no. 6, p. e004072, Jun. 2016.

[6] C.-Y. Lin et al., "Simultaneous amplitude frequency electrogram transformation (SAFE-T) mapping to identify ventricular tachycardia arrhythmogenic potentials in sinus rhythm," JACC Clin. Electrophysiol., vol. 2, no. 4, pp. 459470, 2016.

[7] H. Launer, T. Clark, T. Dewland, C. A. Henrikson, and B. Nazer, "An automated fractionation mapping algorithm for mapping of scar-based ventricular tachycardia," Pacing Clin. Electrophysiol., vol. 42, no. 8, pp. 1133-1140, 2019.

[8] C. Martin et al., "Use of Novel Electrogram 'Lumipoint' Algorithm to Detect Critical Isthmus and Abnormal Potentials for Ablation in Ventricular Tachycardia," JACC Clin. Electrophysiol., vol. 5, Mar. 2019.

[9] B. Campos, M. E. Jauregui, F. E. Marchlinski, S. Dixit, and E. P. Gerstenfeld, "Use of a novel fragmentation map to identify the substrate for ventricular tachycardia in postinfarction cardiomyopathy," Hear. Rhythm, vol. 12, no. 1, pp. 95-103, Jan. 2015.

[10] K. Kuroki et al., "New Substrate-Guided Method of Predicting Slow Conducting Isthmuses of Ventricular Tachycardia: Preliminary Analysis to the Combined Use of Voltage Limit Adjustment and Fast-Fourier Transform Analysis," Circ. Arrhythmia Electrophysiol., vol. 11, p. e005705, Apr. 2018.

[11] G. Baldazzi, M. Orrù, M. Matraxia, G. Viola, and D. Pani, "Automatic Recognition of Ventricular Abnormal Potentials in Intracardiac Electrograms," Comput. Cardiol. 2019, vol. 46, 2019.

Address for correspondence:

Giulia Baldazzi

Department of Electrical and Electronic Engineering,

University of Cagliari

Cagliari, Italy

giulia.baldazzi@unica.it 